\title{
Hispanismes
}

Revue de la Société des Hispanistes Français

\section{« Devaneio e embriaguez duma rapariga » au-delà d'une vague nausée}

«Devaneio e embriaguez duma rapariga» más allá de una vaga náusea «Devaneio e embriaguez duma rapariga» beyond a vague nausea

\section{Ilana Heineberg}

\section{(2) OpenEdition}

Journals

Édition électronique

URL : https://journals.openedition.org/hispanismes/524

DOI : 10.4000/hispanismes.524

ISSN : 2270-0765

Éditeur

Société des Hispanistes Français

Référence électronique

Ilana Heineberg, « « Devaneio e embriaguez duma rapariga » au-delà d'une vague nausée », HispanismeS [En ligne], 15 | 2020, mis en ligne le 01 juin 2020, consulté le 31 juillet 2021. URL : http:// journals.openedition.org/hispanismes/524 ; DOI : https://doi.org/10.4000/hispanismes.524

Ce document a été généré automatiquement le 31 juillet 2021.

Les contenus de cette revue sont mis à disposition selon les termes de la Licence Creative Commons Attribution - Pas d'Utilisation Commerciale - Pas de Modification 4.0 International. 


\title{
« Devaneio e embriaguez duma rapariga » au-delà d'une vague nausée
}

\author{
«Devaneio e embriaguez duma rapariga» más allá de una vaga náusea \\ «Devaneio e embriaguez duma rapariga» beyond a vague nausea
}

Ilana Heineberg

\section{Introduction}

1 Clarice Lispector écrit «Devaneio e embriaguez duma rapariga »en $1954^{1}$, à Washington, où elle vit encore avec son mari, le diplomate Maury Gurgel Valente. La période d'écriture des dernières nouvelles qui seront publiées dans Laços de família (1960) précède la séparation du couple qui devient définitive en 1959 lorsque l'écrivaine s'installe à Rio de Janeiro avec ses deux fils. La nouvelle que nous allons analyser ouvre le recueil et, avec "Preciosidade», est la seule à ne pas connaître de publication préalable ${ }^{2}$.

2 «Devaneio e embriaguez duma rapariga » ne figure pas parmi les plus commentées du livre par la critique. Cela s'explique probablement par le fait que l'héroïne n'atteint pas l'état épiphanique ${ }^{3}$ au même titre qu'Ana, de "Amor », ou Laura, de "Imitação da Rosa », ni l'extase de l'héroïne anonyme de « $O$ búfalo » ${ }^{4}$. L'humour en est aussi un trait marquant, de manière plus évidente que dans d'autres textes de Clarice Lispector ${ }^{5}$. D'ailleurs, il n'est pas anodin que dans son «Explication inutile " Clarice Lispector mette en avant le plaisir que le pastiche de la variante européenne lui a procuré :

Je sais que je me suis tellement amusée que ce fut un réel plaisir de l'écrire. Tout au long de mon travail, j'étais de meilleure humeur qu'au quotidien, et même si les autres ne se sont pas aperçus, j'imitais le parler des Portugais, en m'adonnant apparemment à une expérience langagière. J'ai adoré écrire sur cette Portugaise ${ }^{6}$.

En effet, écrire dans cet autre portugais, le portugais de l'Autre, produit un effet d'étrangeté souvent cherché par Clarice Lispector dans ses associations de mots aussi 
inattendues que poétiques. Les travaux consacrés à la nouvelle se sont souvent intéressés à l'adoption de la norme européenne de l'héroïne qui contamine le discours du narrateur ${ }^{7}$, mais aussi au thème de la conscience de soi ${ }^{8}$, du miroir ${ }^{9}$ et de la rêverie ${ }^{10}$.

Dans notre analyse de "Devaneio e embriaguez duma rapariga ", nous nous demandons d'où vient cette impression d'absence de prétention, de légèreté voire de superficialité de l'expérience narrée dans la nouvelle. Quels éléments textuels nous permettent de la confirmer ou de l'infirmer? Qu'est-ce qu'empêche ou remplace dans l'intrigue de la nouvelle cet état de conscience aigüe de l'existence si souvent présent dans les textes de Clarice Lispector?

5 L'héroïne, la rapariga, est une femme d'origine portugaise de classe moyenne, attachée à sa position sociale mais nostalgique des promesses de sa jeunesse, qui vit dans la rue Riachuelo, au centre de Rio de Janeiro, avec son mari et ses enfants. Les événements de l'intrigue sont constitués essentiellement de dialogues imaginaires, de pensées voire de reproches contre elle-même, de projections de l'héroïne ou par remémorations. La voix du narrateur omniscient et hétérodiégétique épouse la perspective de l'héroïne jusqu'à une imbrication propre au discours indirect libre. Nous avons là ce que Dorrit Cohn appelle le "monologue narrativisé $»^{11}$. De plus, tout comme la rapariga, le narrateur adopte la variante européenne de la langue portugaise ce qui contribue à approfondir cette indifférenciation des deux voix.

Dans le premier mouvement textuel, seule à la maison, «já que os filhos estavam na quinta das titias em Jacarepaguá12 ${ }^{12}$ (p. 10), cette femme au foyer se permet de se laisser aller à la rêverie et de s'assoupir au détriment de la réalisation des tâches ménagères. Puis, brouillant les repères temporels, le texte raconte le dîner, « convite do negociante tão próspero » (p. 12), au restaurant de la Praça Tiradentes, où l'excès de vinho verde la rend à la fois désinvolte, jalouse et tourmentée. Dans un troisième volet, la rapariga est de retour dans sa chambre, avec la sensibilité aiguisée par l'expérience vécue ou remémorée de la sortie au restaurant. Nous pouvons alors nous demander ce qui reste de cette expérience de bovarysme, de rêvasserie et d'ivresse. Notre analyse suivra ces trois mouvements, tout en mettant en avant la constante tension entre - dedans/ dehors, Moi/les autres, le Moi/ les Moi - qui montent in crescendo dans la séquence textuelle pour vraisemblablement se confondre à la fin.

\section{Rêvasseries dans la chambre}

7 Dès son incipit, le texte met en relation la fragmentation du Moi, présente dans l'action de l'héroïne de se regarder dans le miroir, avec le brouillage constitué par l'opposition dehors (la rue) et dedans (la chambre), suggérant par-là que le premier emplit le deuxième: "Pelo quarto parecia-lhe estarem a se cruzar os elétricos, a estremecerem-lhe a imagem refletida. Estava a se pentear vagarosamente diante da penteadeira de três espelhos [...]» (p. 9, nous soulignons). Le bruit arrive aussi par les cris du vendeur de journal qui, tout en renvoyant à la réalité factuelle symbolisée par l'objet journal, provoque une réaction corporelle chez l'héroïne qu'elle ne peut pas identifier : " "A Noite" gritou o jornaleiro ao vento brando da Rua Riachuelo, e alguma cousa arrepiou-se pressagiada ( (p. 9, nous soulignons). La coiffeuse avec ses trois miroirs produit chez le personnage une déformation quelque peu cubiste ${ }^{13}:$ " o roupão aberto deixava aparecerem nos espelhos os seios entrecortados de várias raparigas » (p. 9, nous soulignons). Tout en provoquant un effet évident de clivage, ce 
triptyque annonce trois expressions de l'héroïne qui se confrontent tout au long de la nouvelle : la jeune rapariga du passé ; l'épouse et mère du présent, fière de sa position sociale ; et, enfin, la femme qu'elle aurait pu devenir, une inconnue qui se présente sous la forme d'une femme plus fine et séduisante. Ce bouleversement, qui n'est pas conscient, se traduit dans le texte par le champ lexical du tremblement: de son image projetée dans le miroir (« os espelhos vibravam ora escuros, ora luminosos» (p.9), en passant par le vaisselier - «o estremecimento do guarda-loiça » (p. 10), mais aussi «o sol preso pelas persianas tremia na parede como uma guitarra» (p.10) - jusqu'à atteindre la rue entière: "A Rua Riachuelo sacudia-se ao peso arquejante dos elétricos » (p. 10).

Cette étrange intuition qui ébranle ses certitudes et signale une vie qui aurait pu être différente n'est pourtant ni consciente ni exprimable par l'héroïne. C'est son corps qui parle malgré elle, à l'instar de ce frisson qui est comme un pressage, comme nous l'avons vu plus haut. Elle passe d'un état de joie et de détachement à la colère : « cantou absorta: "quem viu o pardal-zito... passou pela jane-la.. voou pr'além do Mi-nho" - mas colérica, fechou-se dura como um leque » (p. 9). Et vice-versa : « abanava-se impaciente [...]. Punha-se de novo a abanar-se quase a sorrir. Ai, ai suspirou a rir » (p. 9). Il n'est pas anodin que ce soit pendant l'action de s'éventer, qui, saccadée, renvoie aussi à l'agitation et au tremblement, que l'héroïne s'exclame : « Ai, ai vinha da rua como uma borboleta » (p. 10). En se comparant au papillon, c'est encore une fois l'extérieur qui pénètre l'intérieur, dans une apparente insouciance sous-entendue dans l'action de papillonner. Mais le choix du papillon n'est pas dû au hasard; il évoque aussi la métamorphose, c'est-à-dire, ses autres Moi qui se projettent par flashs dans les images des miroirs : «Teve a visão de seu sorriso claro de rapariga ainda nova » (p. 9).

Le dialogue imaginaire de l'héroïne avec un interlocuteur au profil romantique « sorriso galanteador, uns olhos tristes numa dessas caras pálidas que a uma pessoa fazem tanto mal » (p. 10) - devient révélateur à la fois de son propre clivage comme de sa façon de concevoir l'échange verbal avec l'autre. Remarquons qu'elle emploi le substantif "palestra " qui, dans la norme européenne, renvoie plutôt à "conversa amena e informal ${ }^{14}$ ». Le sujet, même s'il doit être " possível e interessante » (p. 10), ne semble pas vraiment central, l'objectif principal du personnage étant clairement de créer un verbiage capable de se montrer sous son meilleur jour, soit ce troisième Moi détaché de la femme au foyer, capable de faire la conversation et d'être séductrice. À un destinataire "galante", elle répond "garrida, de mão à ilharga " (p. 10) dans une évidente mise en scène imaginaire. Il est intéressant de noter la façon dont Clarice Lispector dédouble ici de plusieurs manières non seulement la rapariga mais aussi son interlocuteur. En effet, le narrateur emploie la troisième personne du pluriel dans les verbes introducteurs qui rapportent le discours de ce dernier ("perguntaram-lhe», « insistiram », p. 10), alors que l'héroïne s'adresse à lui à la deuxième personne du singulier («sabes", p. 10). L'usage de la troisième personne du pluriel en portugais renvoie à un sujet indéterminé. L'interlocuteur devient ainsi un et plusieurs, spécifique et vague ce qui multiplie les possibilités d'interprétation de cet échange imaginé par le personnage.

10 En outre, une lecture attentive du dialogue nous permet de déduire que l'héroïne est le sujet même de ce bavardage inventé, qui, il ne faut pas oublier, a lieu devant les trois reflets de la jeune femme dans un moment de rêverie : « sabes quem veio a me procurar cá à casa?», "A Maria Quitéria, homem!» (p.10). Ainsi, de la même manière que, 
d'après Freud, tout rêve traite sans exception du Moi de celui qui rêve ${ }^{15}$, elle serait, dans son affabulation, à la fois interlocutrice et sujet. Cette considération permet, selon nous, de nommer l'héroïne qui était au premier abord anonyme ${ }^{16}$ lorsqu'elle répond en disant le prénom : Maria Quitéria. En posant clairement la question de l'identité ( $\mathrm{E}$ se me permite, quem é esta rapariga?»(p.10, nous soulignons), son interlocuteur imaginaire apparaît « já sem fisionomia » (p. 10), comme s'il commençait à s'évaporer, à l'instar du rêve au moment réveil. La rapariga, en coupant court à cette conversation «"Tu", cortou ela com leve rancor a palestra, que chatura » (p. 10, nous soulignons) -, désigne son locuteur comme étant lui aussi Maria Quitéria. La rapariga, son locuteur et le sujet de la conversation pourraient donc tous être Maria Quitéria. Ce passage, qui chamboule tous les repères énonciatifs, en plus de montrer la fragmentation des Moi de la jeune femme devant le miroir, révèle, par sa construction textuelle, une crise identitaire. La confusion textuelle finit par concerner aussi le lecteur, dernière victime de l'identification problématique de l'héroïne.

11 Par ailleurs, ces clivages font surface à travers les nombreux paradoxes employés pour décrire la jeune femme : «curiosa e entediada » (p. 10), «túrbida e leve» (p. 10), «tão rasa e princesa » (p. 11). Si notre but ici consiste à mettre en avant ce qui est seulement suggéré par la construction textuelle, l'effet de ces contrastes et de l'agitation de l'hérö̈ne reste tout d'abord humoristique. En effet, les oppositions révèlent une jeune femme inconstante, jouant, à son propre insu, un rôle caricatural et faisant des déclarations peu fiables, même lorsqu'elle - ou justement parce qu'elle - se trouve face à elle-même en plein monologue intérieur. Le texte laisse quelques pistes sur le fait que ce jeu de rôle peine à convaincre la rapariga elle-même : "Quem encontrou buscou" disse-se em forma de rifão rimado, o que sempre terminava por parecer alguma verdade » (p. 10, nous soulignons).

12 La condition qui permet à la rapariga de s'échapper ailleurs sans sortir de la chambre résulte, comme nous l'avons vu, de l'absence exceptionnelle de ses enfants et le conséquent allégement des tâches domestiques, ébranlant son rapport au temps et à l'espace, remplaçant le rationnel et le concret par des sensations: "Ai que quarto suculento!» (p.10, nous soulignons). Elle se trouve ainsi dans un état méditatif et d'introspection: «os olhos não se abandonavam» (p. 9), "Os olhos não se despregavam da imagem, o pente trabalhava meditativo » (p. 9, nous soulignons). Au tout début du texte, pendant ce moment narcissique, la présence des autres membres de la famille se fait encore sentir, lorsqu'elle perçoit la chute d'un objet par la fenêtre de l'immeuble : "Se os miúdos e o marido ainda estivessem à casa, já lhe viria à ideia que seria descuido deles» (p. 9). Dans ce premier mouvement textuel, nous observons que le personnage s'efforce d'abord de garder un ancrage dans le réel « aguardava seu próximo pensamento com os olhos abertos " (p. 10), d'où cette tension interne entre une perception plutôt positive du réel et la colère ou l'agacement envers lui.

13 Cependant, après s'être assoupie, le retour envahissant du mari « a voltar do trabalho $\mathrm{e}$ a entrar pelo quatro adentro» (p.10) la réveille, la dérange et provoque une envie d'isolement, allant jusqu'à refuser de jouer son rôle habituel: «Não quis jantar nem sair de seus cuidados, dormiu de novo : o homem lá que se regalasse com as sobras do almoço » (p. 10). Cet étrange état se poursuit le lendemain pouvant alors être nommé : « aproveitou para amanhecer esquisita » (p. 10, nous soulignons). La rapariga peut alors exprimer verbalement son agacement en repoussant son mari «- Larga-te daí!» lorsqu'il essaie de s'approcher d'elle pour l'embrasser, dans un geste qui est clairement 
celui d'un homme exigeant ce qui lui appartient de droit, puis «a ensaiar imediatamente carinho mais eficaz » (p. 11). Lorsque le mari l'interroge sur son état, la rapariga reste coite: «obstinada, não saberia responder, [...] não tinha sequer onde buscar uma resposta " (p.11), mais insiste, de manière plus marquée et agressive, en refusant tout contact: "Não me venhas a rondar como galo velho!» (p. 11). Si elle accepte "surpreendida, lisonjeada » (p. 11) le diagnostic condescendant de maladie du mari - «- Ó rapariga, estás doente» (p.11) -, c'est justement pour prolonger le privilège d'interrompre les tâches domestiques et pouvoir rêvasser, sans qu'elle ait la moindre conscience critique de son rôle subalterne.

Dans ce passage, le lien avec la maladie de Laura, de la nouvelle "Imitação da Rosa », nous paraît évident. Cette dernière rechute à la maison après un séjour à l'hôpital psychiatrique ${ }^{17}$, situation qui semble finalement la libérer de sa vie de femme au foyer. L'héroïne de "Devaneio e embriaguez duma rapariga ", quant à elle, ne s'évade que pendant un temps limité, constitué par les deux événements extraordinaires évoqués par le récit. Contrairement à "Imitação da Rosa", ici, la folie peut être nommée : "doida e delicada no quarto que rodava, que rodava " (p.11, nous soulignons). Puis l'héroïne reprend le diagnostic vague du mari : «Talvez fosse verdade "ó mulher, vê lá se me vais mesmo adoecer!" disse desconfiada. Levou a mão à testa para ver se lhe tinham vindo febres » (p. 12).

Nous pouvons nous interroger alors sur la traduction du mot «devaneio». S'agit-il d'une rêverie ou d'une rêvasserie, comme le proposent les traducteurs Jacques et Teresa Thiériot dans la version française de la nouvelle ${ }^{18}$ ? André Luís Gomes et JeanClaude Miroir ${ }^{19}$, quant à eux, analysent la nouvelle à partir de la poétique de la rêverie de Bachelard. La solution des traducteurs français nous paraît pertinente, car elle a le mérite d'insister sur l'aspect changeant, vague et imprécis des pensées qui habitent la rapariga, allant ainsi dans le sens d'une impression de superficialité laissée par le personnage et de sa faible conscience de ses propres ambivalences et de leurs causes.

Il nous semble également pertinent de rapprocher l'attitude de l'héroïne du bovarysme ${ }^{20}$, non pas en mettant en avant une éventuelle "influence » de Gustave Flaubert sur Clarice Lispector, mais plutôt en le considérant comme une notion qui peut nous aider à préciser l'état de rêvasserie du personnage en question. Maria Quitéria, appelons-la ainsi, contrairement à Emma Bovary, n'est jamais montrée lisant des romans romantiques ni faisant référence à des livres ou à une œuvre de fiction. Néanmoins, elle semble forgée par une réalité patriarcale dominante dans les années 1950 au Brésil qui limitait la femme au rôle d'épouse et de mère, reléguant ainsi le plaisir et le désir au domaine du fantasme. Nous l'avons vu, par exemple, dans la façon dont elle imagine son interlocuteur dans le passage du dialogue imaginaire comme ayant un "sorriso galanteador, olhos tristes numa dessas caras pálidas » (p.10), description qui correspond à l'image mélancolique du mal du siècle comme un moyen justement de s'échapper au carcan patriarcal. Par la suite, c'est elle-même qui semble contaminée par le stéréotype romantique, devenant langoureuse: "Só levantava mesmo para ir à casa de banhos, donde voltava nobre, ofendida " (p. 11, nous soulignons). Le flirt qui n'était que suggéré dans cette fabulation devient plus présent dans les fantasmes de la rapariga une fois qu'elle a repoussé son mari, avec le même dédain qu'Emma le faisait avec Charles. En rêvassant, allongée dans son lit, la jeune femme s'imagine amoureuse, dans une histoire elliptique qui mélange passé et futur : «amava... Estava previamente a amar o homem que um dia ela ia amar. Quem 
sabe lá, isso às vezes acontecia, e sem culpas nem danos para nenhum dos dois » (p. 11, nous soulignons).

La rêvasserie de la rapariga, dans ce premier volet de la nouvelle, rejoint également le bovarysme par le brouillage entre ses fantasmes et la réalité. Chez Flaubert, Emma souhaite vivre comme une héroïne des romans romantiques - amants, richesse, noblesse - refusant les contraintes du réel. Chez Clarice Lispector, l'immersion de Maria Quitéria dans les fantasmes est de courte durée, mais intense, allant jusqu'à contaminer la construction textuelle et à brouiller les frontières entre texte et hors-texte. Ainsi, au fur et à mesure que le récit avance, la voix du narrateur, extérieur à la diégèse, et celle de l'héroïne, interne à la diégèse, se mêlent et l'alternance visible des voix du discours direct devient plus rare. La tension entre maladie (mentale) et santé, comme nous l'avons vu auparavant, peut être aussi mise en rapport avec l'envie, propre au bovarysme, de s'échapper du réel. Enfin, la frontière entre sommeil et veille dans ces deux jours de rêvasserie devient de plus en plus perméable, entraînant la dissolution temporelle : « Nessa noite até dormir fantasticou, fantasticou, por quantos minutos? até que tombou: adormecidona, a ressonar com o marido» (p.12, nous soulignons). Remarquons le néologisme du verbe «fantasticar » employé par Clarice Lispector, dans lequel l'idée de rendre les pensées extraordinaires, contenue dans le mot "fantástico », potentialise le sens de "fantasiar», attesté par les dictionnaires. La confusion temporelle s'accentue ainsi dans la perception de la jeune femme : «A manhã tornou-se uma longa tarde inflada que se tornou noite sem fundo amanhecendo inocente pela casa toda » (p. 12).

18 Ce mouvement textuel se termine par une chute de l'héroïne dans sa réalité des corvées quotidiennes de femme au foyer: "Acordou com o dia atrasado, as batatas por descascar, os miúdos que voltariam à tarde das titias » (p. 12). Pour la deuxième fois, elle s'apostrophe et la voix du personnage n'est pas indiquée par des guillemets, mais seulement par le verbe introducteur du narrateur : "ai que vagabunda que me saíste!, censurou-se curiosa e satisfeita » (p. 12, nous soulignons). Par l'usage de la deuxième personne, le personnage, plus royaliste que le roi, se dédouble et endosse ainsi la perspective du mari et de la société.

\section{Ivresse au restaurant}

Cette partie consacrée à l'épisode de l'ivresse de la rapariga lors d'une sortie au restaurant perturbe les repères temporels du texte. Contrairement à la première partie, pour laquelle nous ne connaissons que la durée de deux journées des rêveries, nous savons que le deuxième passage a lieu samedi soir, justement par ce complément circonstanciel de temps précis et réitéré à trois reprises dans la nouvelle. D’ailleurs, ce volet commence par une adversative - «Mas no sábado à noite» (p.12, nous soulignons) -, qui s'oppose au réveil de l'héroïne dans une journée remplie de tâches ménagères. Il est cependant impossible de le situer dans la temporalité de la nouvelle. Le lecteur se demande comment connecter ces deux événements, faisant ainsi l'expérience de la confusion temporelle du personnage. Quant à la situation spatiale, elle est également précise: la scène de l'ivresse a lieu dans «la tasca da Praça Tiradentes » (p.12), à quelques pas de l'appartement de la rapariga. On passe ainsi de la partie la plus intime du foyer, lieu de la rêverie, à un espace extérieur festif et de 
socialisation, où les fantasmes du personnage deviendront des véritables mises en scènes.

Dans ce passage sans transition du dedans au dehors, la rapariga se projette en tant que personnage mondain. Le soin porté à son apparence trahit le besoin d'affirmer sa position sociale « ela com vestidito novo que se não era cheio d'enfeites era de bom pano superior, desses que lhe iam a durar pela vida afora" (p.12) et de sa jeunesse » Borrachona a não mais poder mas sem perder o brio de rapariga » (p. 12, nous soulignon). L'ivresse n'apparaît pas de manière progressive dans l'extrait qui a lieu dans le restaurant, mais au contraire s'impose d'emblée, dès le titre. Cet état permet à la rapariga de se démarquer de son mari, garant de sa position respectable : «embriagada na Praça Tiradentes, embriagada, mas com o marido ao lado a garanti-la » (p.12). Chef de famille dominateur à la maison, au restaurant, à côté du «negociante tão próspero » (p. 12), «tão mais fino e rico » (p. 12), il doit lui céder son rôle de vieux $\operatorname{coq}^{21}:$ «E se seu marido não estava borracho é que não queria faltar ao respeito ao negociante, e, cheio d'empenho e d'humildade, deixava-lhe, ao outro, cantar de galo » (p. 12, nous soulignons). À l'instar d'Emma Bovary, qui voit d'abord le mariage avec Charles comme une forme d'ascension sociale mais rapidement s'agace face à son manque d'ambition, la rapariga se sent ici à l'opposé de l'état de soumission de son mari envers le commerçant : «procurando dar-lhe palestras, pois que ela não era nenhuma parola d'aldeia e já vivera em Capital»(p. 12, nous soulignons). Elle méprise et se moque de son époux : « 0 que assentava bem para a ocasião fina, mas lhe punha, a ela, uma dessas vontades de rir! um desses desprezos! Olhava o marido metido no fato novo e achava-lhe uma tal piada! » (p. 12, nous soulignons). Rendue euphorique par l'effet de l'alcool, l'héroïne relègue son mari au rôle subalterne qu'elle endosse généralement.

Or cette mise en scène n'est pas sans lien, selon nous, avec l'origine portugaise du couple. Il convient de rappeler que le stéréotype des immigrés portugais au Brésil dans les années 1950, notamment à Rio de Janeiro qui comptait parmi ses habitants de nombreux commerçants portugais, s'illustrait, d'une part, par l'image du riche commerçant explorateur et, d'autre part, par celle de l'immigré pauvre ${ }^{22}$, souvent provenant du Minho. La chanson fredonnée par la rapariga dans sa chambre, dans la première partie de la nouvelle, fait justement référence à cette province historique portugaise et contribue, ainsi, à préciser l'origine régionale et probablement sociale de l'héroïne. On peut déduire facilement le désir de la rapariga de s'approcher plutôt de l'image la plus valorisée socialement de l'immigré, reniant le stéréotype du Portugais primaire et paysan ${ }^{23}$.

En outre, cette attitude a un effet comique lorsque le personnage insiste sur sa position sociale et sur sa préférence pour l'espace urbain, en exagérant largement. Par ailleurs, le texte laisse voir, derrière cette mise en scène, le caractère exceptionnel de cette sortie. Comme Cendrillon qui abandonne ses tâches ménagères pour participer au bal, la rapariga garde au restaurant un souvenir lointain de sa réalité quotidienne : « a alma cotidiana perdida, e que bom perdê-la, e como lembrança dos outros dias apenas as mãos pequenas tão maltratadas ».

Dans ce contexte, l'ivresse est perçue par la rapariga comme un état de fusion capable d'unir ce qui est par nature séparé, confondant les sphères intime et publique, dehors et dedans, affectation et flirt, nourriture et sexe : 
E quando estava embriagada, como num ajantarado farto de domingo, tudo o que pela própria natureza é separado um do outro - cheio de azeite dum lado, homem doutro, terrina dum lado, criado de mesa doutro - unia-se esquisitamente pela própria natureza, e tudo não passava duma sem-vergonhice só, duma marotagem. (p. 12-13, nous soulignons) les deux sens du mot «rapariga» en norme européenne (jeune femme) et brésilienne (prostituée), qui fonctionne comme dénominateur commun de ces deux facettes de Maria Quitéria. Comportant plus de fantasmes que des passages à l'acte, l'ébriété lui permet de se projeter comme femme adultère, marginale, bref la rapariga brésilienne : « e ela agora com os cotovelos sobre a toalha xadrez vermelha e branca da mesa como sobre uma mesa de jogo, profundamente lançada numa vida baixa e revolucionante » (p. 13, nous soulignons).

ar ailleurs, les sensations intérieures provoquées par l'ivresse renvoient à des métaphores de plénitude et légerté : «em verdade, por dentro, estava-se até lá muito bem, era-se aquela nuvem plena a se transladar sem esforço» (p.13, nous soulignons). Nous pouvons noter dans cette phrase le passage à la construction pronominale, qui rend l'agent de l'action indéterminé, vague. Ainsi, ce n'est plus seulement elle, la rapariga, qui accomplit l'action, alors qu'elle est le sujet des phrases précédentes et des subséquentes. Ce choix nous renvoie au dialogue imaginaire de la première partie, où l'interlocuteur est désigné par la troisième personne du pluriel, un autre moyen d'indétermination du sujet dans la syntaxe de la langue portugaise, comme nous l'avons vu. Dans les deux cas, l'usage d'une construction inattendue dans son contexte, dans le but de rendre le sujet de l'action fluctuant, permet de le problématiser et de dénoncer la fragmentation de ce dernier.

Puis, ces sensations évoluent vers une impression de gonflement - «lábios engrossados", "o vinho a inchá-la», "madura e redonda como uma grande vaca»(p.13, nous soulignons). On s'approche alors des images de fertilité qui, curieusement, sont plus en phase avec le monde animal qu'avec l'humain: «Mas as palavras que uma pessoa pronunciava quando estava embriagada era como se estivesse prenhe - palavras apenas na boca, que pouco tinham a ver com o centro secreto que era como uma gravidez. Ai que esquisita que estava » (p. 13, nous soulignons). Dans la distinction faite entre l'adjectif "prenhe» et le substantif "gravidez», le premier terme, employé pour les animaux, renvoie au verbiage de l'ivresse, différente, selon elle, de la "gravidez" qui renverrait à quelque chose d'encore plus profond. Ce gonflement du Moi entraîne une rupture entre le temps intérieur et le temps chronologique, accentuée par l'effet de l'alcool sur les gestes. Ainsi, le narrateur nous indique que "seus gestos eram etapas difíceis até conseguir enfim atingir o paliteiro ", geste qui ne sera accompli qu'à la fin de la scène du restaurant, presque trois pages plus loin.

Peu à peu, l'héroïne semble atteindre cette profondeur, mais sans pouvoir la comprendre entièrement et sans pouvoir l'incorporer à son Moi : « essa gargalhada que estava a sair misteriosamente duma garganta cheia e branca, em resposta à finura do negociante, gargalhada vinda da profundeza daquele sono, e da profundeza daquela segurança de quem tem um corpo » (p. 13, nous soulignons). L'héroïne sent ainsi, au plus profond d'elle-même, une présence qu'elle perçoit comme étrangère justement parce que celle-ci est encore endormie. De plus, à ce stade, ce n'est plus son mari qui la protège (« seu marido ao lado a garanti-la », p. 12), mais son propre corps (« segurança 
de quem tem um corpo ", p. 13). Elle assiste à sa transformation en se dédoublant encore une fois: "Palestrava e ouvia com curiosidade o que ela mesma estava a responder ao negociante abastado [...]. Ouvia intrigada e deslumbrada o que ela mesma estava a responder: o que dissesse neste estado valeria para o futuro em augúrio » (p. 14, nous soulignons). Cette perception de soi comme clairvoyante dialogue avec l'image précédente du nuage, mais aussi avec celle du projecteur qui offre une perspective à la fois hors d'elle mais aussi d'en haut : " Um holofote enquanto se dorme que percorre a madrugada - tal era a sua embriaguez errando lenta pelas alturas » (p. 14).

Lors de cette expérience qui est à la fois une sortie et une plongée en soi, la blancheur domine la description du personnage: "dentes brancos»(p.13, nous soulignons), "garganta cheia e branca" (p.13, nous soulignons) et "carne alva" (p.13, nous soulignons); elle est présente aussi dans la lumière du projecteur, comme l'indication d'une connaissance vague ${ }^{24}$. Mais, lorsque l'image de la langouste est évoquée, la blancheur se confond dans le texte avec la douceur et la délicatesse de la chair. Comparée à cette nourriture fine, la rapariga crée un jeu sensuel avec ce riche commerçant, perçu par elle comme nous l'avons vu, comme un homme plein de finesse. La douceur devient très vite paradoxale: « $\mathrm{E}$ aquela vontade de se sentir mal para aprofundar a doçura em bem ruim. E aquela maldadezita de quem tem um corpo » (p. 13, nous soulignons). En effet, elle refuse rapidement ce rôle : « já agora ela não era lagosta, era um duro signo: escorpião. Pois que nascera em novembro» (p. 14). Cette métamorphose de langouste en scorpion contient, d'une part, une certaine continuité par la forme courbée de l'un et de l'autre et, d'autre part, une rupture, de passer de victime à prédateur venimeux, d'aller de la lumière au souterrain. Lorsqu'elle évoque son signe du zodiaque, la rapariga en retient seulement la dureté, alors qu'il symbolise également la «dialectique de la destruction et de la création, de la mort et de la renaissance, de la damnation et de la rédemption ${ }^{25}$.

Cet aspect apparait par la suite lors la contemplation d'un tableau de nature morte («quadro de uvas e peras e peixe morto brilhando nas escamas», p. 14). Le plaisir procuré par le tableau révèle l'affinité de l'héroïne avec l'activité artistique: «que sensibilidade », le mot " sensibilidade » étant répété quatre fois. La scène n'est pas sans humour, car cette réaction cathartique n'a pas lieu dans un endroit où l'art est sacralisé, comme un musée, mais dans un restaurant, où les tableaux sont généralement des reproductions dans un simple but décoratif. En outre, il s'agit d'une nature morte, genre considéré comme mineur dans la peinture. Cette situation révèle donc les velléités de sensibilité artistique de la rapariga présentées comme incompatibles avec son rôle domestique. L'art se retrouve, à l'instar de l'héroïne, décalé de son rôle sacralisant pour se retrouver dans un restaurant. À noter que ces décalages - du personnage et de l'art - sont représentés par le biais de l'exagération : « quando olhava o quadro tão bem pintado do restaurante ficava logo com sensibilidade artística » (p. 14, nous soulignons).

Cette sensibilité - même si elle est décalée et déplacée - lui permet de ressentir aussi des sensations proches de la douleur, que nous pouvons associer à la connaissance de sa médiocre situation: "incomodava sem sem dolorosa, como uma unha quebrada " (p. 14). La comparaison prosaïque avec l'ongle cassé la remet à nouveau en décalage avec une vision sacralisée de l'art. 
31 Puis, ce désir d'évasion provoqué par la contemplation du tableau est confronté à la vie domestique de la rapariga révélant par là le vrai conflit du personnage. L'héroïne se considère d'abord dans une situation privilégiée : " podia permitir-se ao luxo de tornar ainda mais sensível, ainda podia ir mais adiante: porque era protegida por uma situação, protegida como toda a gente que atingiu uma posição na vida » (p. 14, nous soulignons). Dans ce raisonnement, c'est le mariage et sa situation socio-économique confortable qui lui permettraient de se laisser emporter par l'art. Puis, elle s'exclame : «Como sou infeliz, minha mãe! » affirmant que les liens familiaux sont ressentis à ce moment-là plutôt comme une contrainte. Pour sortir de cette prise de conscience gênante, la solution pour la rapariga serait de « emborrachar-se ainda mais, contando que não perdesse o brio » (p.14). On perçoit clairement le parallèle qui se construit entre, d'une part, cette "sensibilidade artística », l'exaltation devant ce tableau du restaurant, et l'ivresse, de l'autre.

Dérangée par cette impossibilité de concilier sa sensibilité artistique avec sa condition de femme au foyer, le personnage déplace son regard du tableau vers les personnes qui se trouvent au restaurant. Cette vision panoramique - celle du projecteur - lui procure un sentiment de mépris, très différent des sensations provoquées par le tableau : «Que desprezo pelas pessoas secas do restaurante » (p. 14, nous soulignons). Notons que ces «personnes sèches » du restaurant peuvent évoquer la maigreur en portugais, mais s'opposent aussi à l'adjectif « humide ", qui renvoie à la chair, aux sentiments. On voit que la rapariga cherche aussi des âmes sœurs, des personnes qui puissent sentir comme elle cette "sensibilidade artística" (p.14): "homem que fosse homem a valer, que fosse triste mesmo"(p.14, nous soulignons). La tristesse est ici en lien avec la sensation de gêne provoquée par le tableau. Les adjectifs " grossa e pesada, generosa » (p.14), qui renvoient dans toute la nouvelle à sa caractérisation en tant que matrone respectée, gagnent alors le sens d'une force artistique, s'opposant à " pessoas secas ».

Lorsque le regard de la rapariga se pose sur la jeune femme qu'elle avait aperçue dès son entrée dans le restaurant, une nouvelle sensibilité est éveillée, provoquant un deuxième moment d'exaltation intérieure, traduit dans le texte par « aquela rapariga que já d'entrada, lhe fizera subir a mostarda ao nariz» (p.15). Différente de la sensibilité artistique provoquée par le tableau, ici elle se sent irritée, agacée, dérangée par la jeune femme "toda cheia de chapéus e d'ornatos, loira como um escudo falso » (p. 15). L'héroïne est clairement jalouse de cette jeune femme qui compose avec elle un jeu de miroir inversé.

34 Se montrant jusqu'à présent plutôt fière de son apparence de matrone - elle est décrite comme étant forte, généreuse -, la rapariga remarque le corps mince de sa rivale : « cinturita fina » (p. 15), « que nem roliça era, era chata de peito » (p. 15). Tout en cette femme semble renvoyer au bon goût (" rico chapéu bem posto ", p. 15) et à une finesse discrète que la rapariga associe tantôt à une modestie presque religieuse: "toda santarrona » (p. 15), " ostentar aquele ar de santa » (p.15), "aproveitasse a beatice » (p.15), «modesta» (p.15); tantôt à des traits aristocratiques: «e que não lhe entornasse a fidalguia na sopa! » (p. 15, nous soulignons), «fina » (p. 15), « a fidalguita de chapéu » (p. 15, nous soulignons). L'attention que réserve le serveur à l'autre femme est également perçue avec jalousie.

35 L'aspect physique et l'apparence soignée de la jeune femme au chapeau sont décrits comme exagérés («loira como um escudo falso, p. 15)», voire théâtraux. La dévalorisation de la rivale se fait ainsi par la transformation de son apparence en indice 
d'incompatibilité avec ce qu'elle, par conformisme, considère comme un destin de femme digne, le mariage et la maternité : « vai ver que não era capaz de parir-lhe, ao seu homem, um filho ». Quant à l'aspect chic et discret de la femme, cela devient un indice d'une certaine luxure - «As mais santazitas eram as que mais cheias estavam de patifaria » (p. 15, nous soulignons) - ou bien d'une vulgarité bien cachée - «E vai ver que, com todos os seus chapéus, não passava duma vendeira d'hortaliça e se fazer passar por grande dama » (p. 15, nous soulignons).

Le seul avantage que l'héroïne semble avoir face à sa rivale reste son statut de femme mariée et de mère, qu'elle avait montré comme ambivalent auparavant : son garde-fou face à sa sensibilité artistique, mais aussi une limite à une vie plus exaltée. La crise de la rapariga arrive ici à son paroxysme lorsqu'elle exprime son envie de frapper l'autre : « crescera-lhe a vontade de ir e d'encher-lhe, à cara de santa loira da rapariga, uns bons sopapos, a fidalguita de chapéu.» (p. 15). Suite à cette agression physique imaginaire, soulagée, la rapariga est prête désormais à avouer son humiliation : "a cabeça agora parecia-lhe nua » (p. 15), mais pour mieux s'emporter à nouveau dans une avalanche d'insultes : «E se pensas que t'invejo e ao teu peito chato, fica a saber que me ralo, que bem me ralo de teus chapéus. A patifas sem brio como tu, a se fazerem de rogadas, eu lhas encho de sopapos. » (p. 15)

Ce n'est certainement pas anodin que le narrateur se réfère à la crise que nous venons d'analyser par l'oxymore «sagrada cólera » (p. 15). Cela montre bien l'opposition entre sacré et profane présente dans tout cet extrait entre, d'un côté, le confort et la sécurité de son rôle de femme et de mère, et, de l'autre, l'appel de la liberté, qu'elle soit artistique ou féminine.

\section{Chambre et chair}

Le retour à l'espace de la chambre remet le lecteur dans la peau du personnage ivre qui se retrouve déplacé dans un autre lieu sans savoir comment. La transposition du restaurant à la maison se fait donc de manière elliptique et, à l'instar du mouvement inverse, commence par une conjonction adversative : «Mas finalmente a dificuldade de chegar em casa desapareceu: remexia-se agora dentro da realidade familiar de seu quarto » (p. 16). Les oppositions dehors-dedans, société-famille et social-intime sortent ainsi renforcées. "A chinela a se balançar no pé ", signe de l'impatience de l'héroïne, indique sa difficulté de se sentir apaisée à cause de son impossibilité à concilier ses désirs contradictoires.

Lorsque la rapariga décide d'entrefermer les yeux, la chambre se transforme en une extension de son corps où tous les éléments deviennent sensibles: "tudo ficou de carne, o pé da cama de carne, a janela de carne, na cadeira o fato de carne que o marido jogara, e tudo quase doía » (p. 16). On peut constater ici que la conscience de l'héroïne s'étend aussi, atteignant un état de grande sensibilité métaphorisée justement par l'omniprésence de la chair ${ }^{26}$. Cette lecture est corroborée par le fait que la rapariga se perçoit en expansion, comme si elle-même devenait tout un univers à être explorer, mais qui garde une grande part de mystère : «E ela cada vez maior, vascilante, túmida, gigantesca. Se conseguisse chegar mais perto de si mesma, ver-se-ia inda maior. Cada braço seu poderia ser percorrido por uma pessoa, na ignorância que se tratava de um braço, e em cada olho podia-se-lhe mergulhar dentro e nadar sem saber que era um olho » (p. 16, nous soulignons). Remarquons cependant que l'impossibilité d'auto- 
connaissance est posée par l'usage de l'imparfait du subjonctif ("se conseguisse ») suivi du conditionnel (futuro do pretérito), montrant qu'elle n'est pas arrivée à atteindre cet état.

Si la chair renvoie ici à la sensibilité, à l'humanité, le mot nous permet également d'établir des liens avec la description de l'héroïne comme une femme « toute en chair ", en opposition à cette autre femme mince, élégante, belle et libre qu'elle voit au restaurant, mais aussi, auparavant dans la chambre, dans son image reflétée par le miroir de sa coiffeuse. La chair passe ainsi d'un rembourrage censé assurer une assise sociale et une sécurité affective à une matière profonde et intime.

$41 \mathrm{Si}$, dans les premières lignes du passage étudié, le tableau la gênait au même titre que son ongle cassé, dans ce troisième mouvement, la douleur est universelle, omniprésente et se dévoile in crescendo : «e tudo quase doía » (p. 16), « e ao redor tudo a doer um pouco » (p. 16, nous soulignons), «As coisas feitas de carne com nevralgia » (p. 16), allant d'une douleur vague à une douleur localisée et aiguë qui est le propre de la névralgie. On peut remarquer que la douleur n'est pas exclusivement celle du personnage, mais une douleur du monde qui, de ce fait, devient diffuse. Une fois que l'héroïne atteint ce paroxysme de sensibilité, de douleur et de communion, elle finit par expliquer son attitude par une petite maladie passagère, qui ressemble beaucoup à celle que son mari avait diagnostiqué pour justifier son état au début de la nouvelle : « Fora o friozito que a tomara ao sair da casa de pasto » (p. 16). Elle revient par cette explication à ce qu'on attend d'elle : "conformada e cética » (p. 16). Mais sceptique par rapport à quoi ? Par rapport à l'expérience de sensibilité exacerbée vécue ou à l'imminence de revenir à son rôle habituel ?

L'hérö̈ne se trouve, à la fin de la nouvelle, entre l'ivresse et le retour dans son quotidien; elle vit cette transition de manière intense mais hésitante. Craignant que « nesse momento lhe estavam a acontecer cousas que só mais tarde iriam a doer mesmo e a valer » (p. 16), elle décide d'affronter la réalité en ouvrant les yeux. Quelle surprise : «tudo ficou menor e mais nítido, embora sem nenhuma dor. Tudo, no fundo, estava igual, só que menor e familiar» (p. 16). En effet, le réel est souvent moins angoissant que ce que l'on se représente la nuit, mais il devient pour cela extrêmement ennuyeux pour la rapariga. Une énumération de qualificatifs permet de saisir la passivité qui envahit l'héroïne : «absorta », "resignada », « com delicadeza de quem espera sentado que outro acorde ", " paciente », " obediente ». Ainsi, par cette attitude, elle devient lectrice de sa vie: «Ai, palavras, palavras, objetos do quarto alinhados em ordem de palavras, a formarem aquelas frases turvas e maçantes que quem souber ler, lerá. Aborrecimento, aborrecimento, ai que chatura... Que maçada» (p.16-17). Ce passage montre que la rapariga, capable de voir des mots (des référents) dans les objets qui auparavant étaient constitués de matière sensible - la chair -, ne peut pas, en revanche, ni saisir, ni transmettre leurs sens. La sensation d'ennui découle, selon nous, justement de cette impossibilité: "Ai, é uma tal cousa que se me dá que nem bem sei dizer» (p. 17, nous soulignons).

De ce fait, elle semble coincée entre l'ivresse et le quotidien, entre le dehors et le dedans, entre les mots et le sens. Cela apparaît dans le texte à travers plusieurs exemples d'injonctions contradictoires, pouvant concerner un simple appel au divertissement: "Mesa! gritou-lhe o mundo. Mas ela nem sequer a responder-lhe " (p. 17). Cet appel fait écho au cri du vendeur du journal A Noite, au tout début de la nouvelle. Comme nous l'avons analysé, les sons de la rue pénétraient alors la chambre 
comme un appel à la rêverie, alors qu'ici le monde extérieur, plus vaste que sa rue, résonne encore dans les souvenirs des moments passés au restaurant. De même, lorsque la rapariga se reproche ses excès au restaurant: " "Empanturras-te e eu que pague o pato", disse-se melancólica » (p. 16), elle ressent un clivage entre son Moi qui s'amuse et celui qui subit les conséquences, enfin, son Moi extérieur et celui du foyer. Ce tiraillement apparaît également lorsque le narrateur se remet à lister des qualificatifs qui, cette fois, révèlent les contradictions du personnage qui a fait l'expérience de sortir de soi grâce à l'ivresse : «desiludida, resignada, empanturrada, casada, contente, a vaga náusea » (p.17). Cette nausée que le narrateur tient à qualifier de vague, liée certes à l'excès de nourriture («empanturrada») et de vinho verde, constitue l'expression de cette impossibilité de trouver un compromis entre dehors et dedans ${ }^{27}$. Différente, par exemple, de la nausée ${ }^{28}$ ressentie par Ana, dans la nouvelle "Amor", la nausée de la rapariga ne relève ni de l'absurdité de l'existence, ni de l'extase mystique, mais, selon nous, de l'intuition d'une autre vie possible que le personnage n'est pas encore prêt à embrasser.

Notre lecture se confirme par la sensation de surdité qui suit la nausée, que l'héroïne combat en se tapant l'oreille du plat de la main ce qui provoque l'effet inverse :

encheu-se-lhe o ouvido de um rumor de elevador, a vida de repente sonora e aumentada nos menores movimentos. Das duas uma: estava surda ou a ouvir demais - reagiu a essa nova solicitação maliciosa e incômoda, com um suspiro de saciedade conformada. (p. 17, nous soulignons)

Ce passage semble symboliser la conscience de la rapariga de sa situation d'épouse et mère dans une société patriarcale qui ne lui laisse pas un grand choix de destins. La sensation de "satiété résignée » suit ainsi à la "vague nausée ", signifiant que l'excès $\mathrm{du}$ restaurant lui a permis d'échapper au quotidien pour mieux y retourner. La certitude du bien-être apportée par l'acceptation de son destin lui vient après un flashback de deux scènes de la soirée, lorsque l'héroïne attribue cette sensation de " presque nausée », non plus à l'ivresse, mais à sa vie familiale : « Havia certas cousas boas porque eram quase nauseantes: o ruído como de elevador no sangue, enquanto o homem roncava ao lado, os filhos gorditos empilhados no outro quarto a dormirem, os desgraçadinhos » (p. 17-18, nous soulignons).

Il est intéressant de s'arrêter sur la scène du restaurant qui revient à l'esprit de l'héroïne au moment du coucher. Il ne s'agit plus de son ivresse ou de l'excès de nourriture, mais du fait que celui qu'elle appelle maintenant, non sans ironie, le "protecteur de son mari » lui glisse un pied contre le sien sous la table. "Porque calhara ou de propósito?» (p. 17), se demande-t-elle dans sa chambre. Jusqu'à présent les rêvasseries se produisaient à partir de son propre clivage, alors que, là, c'est l'attitude de cet homme "que era lá bem interessante» (p.17) qui est interrogée. Son attirance est-elle réciproque ?, se demande au fond la rapariga, s'embarquant à nouveau dans le bovarysme. Clarice Lispector répond avec un autre souvenir, qui remplace l'arsenic de Madame Bovary par une bonne dose de dérision ${ }^{29}$ : «E quando no seu decote redondo em plena Praça Tiradentes!, pensou ela a abanar a cabeça incrédula - a mosca se lhe pousara na pele nua? Ai que malícia » (p. 17).

La nouvelle se finit par un retour en force de la femme au foyer et juxtapose mélancolie, résignation et colère. En effet, la rapariga constate plusieurs fois sa tristesse et son impuissance: "Era a tristeza» (p.18), «Ai que tristeza. Que é que se há de fazer. » (p. 18). Ses pieds qui, plus haut, trahissaient son impatience, s'apaisent et se 
conforment à la situation: «Os dedos do pé a brincarem com a chinela » (p. 18). De manière inattendue et disproportionnée pour le lecteur, produisant ainsi un effet comique, la rapariga se met en colère et annonce un grand nettoyage de la maison qui se présente contradictoirement comme un rituel de purification et de vengeance: "Aquela sua casa havia de ver! dar-lhe-ia um esfregaço com água e sabão que se lhe arrancariam as sujidades todas! a casa havia de ver! ameaçou ela colérica.» (p. 18, nous soulignons). Personnifiée ainsi, la maison, qui tiendrait à sa saleté comme l'héroïne malgré elle à sa dose de divertissement, se confond avec la maitresse de maison lorsque celle-ci s'exclame : "Que relaxada e preguiçosa que me saíste» (p. 18). L'effet comique ne se produit pas seulement de l'éventuelle légèreté de la situation l'ivresse de la femme au foyer qui repousse le grand ménage au lendemain lorsqu'elle sera «bem das pernas ». Il vient surtout du fait que le personnage n'arrive pas à se rendre compte à quel point elle incarne, plus que son mari, qui ronfle à côté d'elle, les règles patriarcales de la société.

Entre reproches et ressaisissements qui renforcent l'autoperception dégradée de cette maîtresse de maison, il n'est pas anodin qu'elle sente sa féminité revenir avec force lorsqu'elle pense une dernière fois à la soirée : «Ai que se sentia tão bem, tão áspera, como se ainda tivesse a ter leite nas mamas, tão forte. Quando o amigo do marido a viu tão bonita e gorda ficou logo com respeito por ela » (p. 18). Les images de fertilité et de gestation, donc de jeunesse, se confondent dans ce passage avec son aspect de matrone, la rendant attirante par ce qu'elle est à présent. Notons que la nausée provoquée par le bonheur familial, évoquée plus haut, n'est pas sans lien avec l'idée de gestation, car souvent présente en début de celle-ci. Par cette juxtaposition désordonnée de ressentis liés à sa féminité - sensualité, respect, emprise des tâches ménagères -, la rapariga rassemble ses multiples Moi avant de finir sa journée tandis que le narrateur insiste sur le mot « resignada».

Enfin, l'hérö̈ne regarde la lune faisant une avec ce satellite sans lumière propre, simple reflet du soleil qu'elle découvre, étonnée, à sa fenêtre : « A lua alta e amarela a deslizar pelo céu, a coitadita. A deslizar, a deslizar... Alta, alta »(p. 18, nous soulignons). Notons que l'adjectif " alto", en argot brésilien, peut désigner également une personne ivre ${ }^{30}$. Or, auparavant au restaurant, la rapariga s'était identifiée par son ébriété à un projecteur dans les hauteurs: «Um holofote enquanto se dorme que percorre a madrugada - tal era a sua embriaguez errando lenta pelas alturas» (p. 14, nous soulignons). La perception de la lune, comme celle que nous avons de l'héroïne, change constamment - croissant, décroissant, disparaissant -, d'où l'association de cet astre aux rythmes biologiques, à la femme et à la fécondité ${ }^{31}$. Observer la lune revient ainsi à se remettre devant les trois miroirs et à se voir multiple et paradoxale, ce qui provoque cette réaction ambivalente adressée à la fois à elle-même et à la lune : "a grosseria explodiu-lhe em súbito amor: cadela, disse a rir » (p. 18).

\section{Conclusion}

51 Nous avons analysé « Devaneio e embriaguez duma rapariga » en nous interrogeant sur les éléments textuels qui conduisent la critique et les lecteurs de Clarice Lispector à la considérer comme plus légère et parfois prendre cette nouvelle moins au sérieux que d'autres de Laços de família. Sa place en ouverture du recueil, formant, d'une part, une paire antithétique avec la cathartique «O Búfalo » qui le clôt et, d'autre part, anticipant 
la thématique de « Amor » et «Imitação da rosa », ainsi que sa dimension humoristique constituent certainement une première confirmation de cette lecture.

Comme le montre le témoignage de Clarice Lispector, l'aspect ludique, présent dans la construction de la voix du personnage d'origine portugaise, représente une dimension importante de la nouvelle. Nous avons vu que le décalage entre les deux variantes peut contribuer à un effet comique lorsque les sens de certains mots sont contradictoires par exemple rapariga et palestrar. Il n'est pas anodin que cet effet comique tombe justement sur l'ancien colonisateur, représenté dans la nouvelle par le stéréotype de l'immigré, incarné par le mari de l'héroïne. Ce personnage est subalterne par rapport au commerçant prospère qui constitue l'objet de désir de la rapariga. Ainsi, on peut voir dans cette représentation, bien plus qu'un jeu avec la langue du colonisateur : un jeu avec les relations de pouvoir entre femmes et hommes et entre classes sociales qui ne sont pas sans lien avec la colonisation.

Par ailleurs, l'effet d'étrangeté de la norme portugaise contribue à accentuer les ambivalences de l'héroïne : fière de sa position économique confortable, de son mariage et de ses enfants, elle désire, par ailleurs, sortir de sa routine, être libre et plaire aux hommes tout en se servant justement de ces atouts. Ainsi, ce désir d'évasion s'exprime à travers une forme de bovarysme. Dans ses rêvasseries romantiques, elle s'imagine parlant d'elle-même avec un homme, se revoit jeune et repousse son mari lors qu'il essaye de la toucher. Puis, ivre au restaurant, elle développe ce qu'elle appelle sa sensibilité artistique, jalouse d'une femme plus jeune, mince et raffinée, et flirte avec le protecteur de son mari. Composé de fantasmes sans véritables passages à l'acte, son bovarysme met à nu les contradictions du personnage tout en lui permettant de continuer à supporter sa routine.

54 Malgré la dimension comique du personnage, le texte reprend de nombreux motifs présents dans l'œuvre de Clarice Lispector liés à la réflexion sur l'existence. Au début de la nouvelle, le miroir fragmente l'identité de l'héroïne, laissant entrevoir, à travers les reflets de ses autres Moi, ses désirs et ses frustrations. Après avoir plongé son regard dans elle-même, elle examine les personnes autour - le mari, le commerçant, la femme avec le chapeau -, selon la perspective surélevée du projecteur, mais qui ne lui permet pas de sortir véritablement d'elle-même et de prendre du recul. Si le protagoniste entraîne le lecteur dans une confusion temporelle et spatiale propre à ses états de rêvasserie et d'ivresse, la perspective narrative et la construction textuelle permettent à ce dernier de déceler certaines relations de domination sociale - le mari/ la rapariga, le mari/le commerçant prospère, la femme mariée/femme séduisante - qui restent cependant invisibles pour le personnage.

Finalement, le texte met en évidence une dilution des frontières entre réel et fantasme, dedans (la maison, Moi, la famille) et dehors (la rue, les autres, la société) qui appelle finalement à l'interprétation. De même, l'expérience d'une sensibilité extrême fait que la rapariga perçoit la chambre «en chair » et a une intuition d'un sens profond de la vie derrière toutes ses sensations. Contrairement à d'autres personnages de Clarice Lispector qui s'enfoncent plus dans leur introspection et s'interrogent sur la manière de l'exprimer tout en le disant déjà, la rapariga n'atteint pas l'épiphanie faite de pur langage. Toutefois, avec sa "vague nausée ", elle préfigure cet état, faisant de la position de la nouvelle au seuil de Laços de família, une annonce des textes à venir. L'héroïne, qui a l'intuition qu'elle n'a pas encore atteint «le centre secret qui était 
comme une grossesse », laisse au lecteur le soin de poursuivre sa tâche : «celui qui sait lire, lira... »

\section{BIBLIOGRAPHIE}

Boaventura DE SOUSA SANTOS, « Entre Próspero e Caliban. Colonialismo, Pós-colonialismo e interidentidade », Novos Estudos CEBRAP, nº 66, (juillet 2003).

Olga BORELl, A escritura de Clarice Lispector, Petrópolis, Vozes, 1979.

Nádia Battela Gotlib, Clarice, uma vida que se conta, São Paulo, Editora Ática, 1995.

Jean CHEVALIER et Alain GHEERBRANT, Dictionnaire des symboles, Paris, Robert Laffon, Jupiter, 1982.

Dorrit COHN, La transparence intérieure, Paris, Seuil, 1989.

Dicionário Houaiss da língua portuguesa, Rio de Janeiro, Editora Objetiva, 2001.

Dicionário infopédia da Língua Portuguesa, Porto, Porto Editora, 2003-2020 [disponible le 19/07/2020] <URL : https://www.infopedia.pt/dicionarios/lingua-portuguesa/palestra>.

Sigmund FREUD, L'interprétation du rêve, traduction et présentation par Jean-Pierre Lefebvre, Paris, Seuil, 2010.

Jules DE GAUlTIER, Le Bovarysme, suivi d'une étude de Per Buvik, Le Principe bovaryque, Paris, Presses de l'Université Paris-Sorbonne, 2006.

Moacyr GODOY MOREIRA, Linguagem e melancolia em Laços de família: histórias feitas de muitas histórias, [dissertação de mestrado], São Paulo, USP/FFLCH, 2007.

Delphine JAYOT, « Portrait(s) d’Emma : une femme “écrite" aux femmes décrites », Anne Herschberg Pierrot (dir.), Flaubert, éthique et esthétique, Saint-Denis, Presses universitaires de Vincennes, p. 81-94.

Vassilis KAPSAMBELIS, « Bovarysme », Philippe Zawieja (dir.), Dictionnaire de la fatigue, Paris, Librairie Droz, 2016.

Valérie C. LASTINGER, « Humour in a new reading of Clarice Lispector », Hispania, 72, 1 (1989), p. 130-137.

Yvan LECLREC, « Bovarysme, histoire d'une notion », Yvan Leclerc et Nicole Terrien. Le bovarysme et la littérature de langue anglaise, Mont-Saint-Aignan, Presses universitaires de Rouen et du Havre, 2004, p. 3-13 [disponible le 07/07/2020] <URL : http://books.openedition.org/purh/7271>.

Naomi LINDSTROM, «A Feminist Discourse Analysis of Clarice Lispector's "Daydreams of a Drunken Housewife" ", Latin American Literary Review, vol. 9, n¹9 (automne-hiver 1981), p. 7-16 [disponible le 13/05/2020], <URL : https://www.jstor.org/stable/20119271>.

Clarice LISPECTOR, Liens de famille, traduit du brésilien par Jacques et Teresa Thiériot, Paris, Éditions des femmes Antoinette Fouque, 1989.

Clarice LISPECTOR, Laços de familia, Rio de Janeiro, 2009 [1960]. 
Clarice LISPECTOR, « Appendice : l'explication inutile », Nouvelles [édition complète établie par Benjamin Moser], Paris, Éditions des femmes Antoinette Fouque, 2017.

Jean-Claude MIROIR et André Luís GOMES, « Poética e devaneio no processo criativo de Clarice Lispector », Fernanda Coutinho et Vera Moraes (org.), Clarices: uma homenagem (90 anos de nascimento, 50 anos de Laços de família), Fortaleza, Edições UFC, 2012, p. 35-55.

Benjamin MOSER, Clarice, uma biografia, tradução de José Geraldo Couto, São Paulo, CosacNaify, 2011

Teresa Cristina MONTERO FERREIRA, Eu sou uma pergunta. Uma biografia de Clarice Lispector, Rio de Janeiro, Rocco, 1999.

Benedito NUNES, O drama da linguagem, São Paulo, Ática, 1995.

Maria Luísa NUNES, « Modes in Clarice Lispector's Laços de familia: The rendering of Consciousness ", Luso-brazilian review, vol. 14, n² (1977), p. 174-184.

Cláudia PAZOS ALONSO, « Do centro e da periferia: uma releitura de Laços de família », Veredas, vol. 3, t. 1, (décembre 2000), p. 287-299.

Regina PONTIERI, «Visões da alteridade: Clarice Lispector e Maurice Merleau-Ponty », Revista USP, n44, (déc-janv-févr, 1999-2000), p. 330-334.

André RAMOS GARCíA, « Decisiones traductoras y efectos en la lectura: estudio de dos traducciones al español de "Devaneio e embriaguez duma rapariga" de Clarice Lispector », Cadernos de Tradução, vol. 40, n¹, 2020, p. 168-187 [disponible le 17/07/2020] <URL : https:// periodicos.ufsc.br/index.php/traducao/article/view/2175-7968.2020v40n1p168 >.

Affonso ROMANO DE SANT'ANNA, "O ritual epifânico do texto », Clarice Lispector, A paixão segundo G.H., [édition critique de Benedito Nunes], Madrid ; Paris ; México ; Buenos Aires ; São Paulo ; Lima ; Guatemala ; San José de Costa Rica ; Santiago de Chile, ALCCA XX, 1997.

Robert ROWLAND, « Manuéis et Joaquins: a cultura brasileira e os portugueses », Etnográfica, Vol. V (1) (2001), p. 157-172.

Gabriela RUGGIERO NOR, Imagens de espelho em Clarice Lispector: entre reflexos e passagens [dissertação de Mestrado], São Paulo, USP/FFLCH, 2012.

Ernani TERRA, « O enunciador em um conto de Clarice Lispector », Entretextos, vol. 20, $\mathrm{n}^{\circ} 1$ (janvierjuillet 2020), p. 241-255.

Paloma VIDAL, Involontaires de la patrie : Clarice Lispector et les minorités, une lecture de la nouvelle «Amor », [conférence], Bordeaux, Université Bordeaux Montaigne, le 14 avril 2019.

\section{NOTES}

1. Nádia Battela gotlib, Clarice, uma vida que se conta, São Paulo, Editora Ática, 1995, p. 269.

2. Teresa Cristina MONTERO FERREIRA, Eu sou uma pergunta. Uma biografia de Clarice Lispector, Rio de Janeiro, Rocco, 1999, p.211. En effet, «Mistério de São Cristóvão», «Os laços de família», "Começos de uma fortuna », «Amor », «Uma galinha » et « O jantar » sont publiées en 1952 dans le recueil Alguns contos. Les autres nouvelles de Laços de família paraissent avant la sortie du livre dans le magazine hebdomadaire Senhor.

Pour ce qui est de la biobibliographie de Clarice Lispector, cf. également : Benjamin MOSER, Clarice, uma biografia, tradução de José Geraldo Couto, São Paulo, Cosac Naify, 2011, p. 346-348 ; Nádia 
BATTELA GOTLIB, op. cit., p. 269-273 et p. 308-330; INTITUTO MOREIRA SALLES, Cadernos de Literatura Brasileira: Clarice Lispector, São Paulo, Instituto Moreira Salles, 2004, p. 23-27.

3. L'épiphanie est comprise comme une révélation, un moment lumineux, suite à une expérience banale et routinière. Affonso Romano de Sant'Anna explique que l'épiphanie peut être comprise dans un sens mystique-religieux : l'apparition d'une divinité et une manifestation spirituelle, le plus souvent l'apparition du Christ. En littérature : «é a percepção de uma realidade atordoante quando os objetos mais simples, os gestos mais banais e as situações mais cotidianas comportam iluminação súbita da consciência dos figurantes, e a grandiosidade do êxtase pouco tem a ver com o elemento prosaico em que se inscreve o personagem ", Affonso ROMANO DE SANT'ANNA, "O ritual epifânico do texto ", Clarice Lispector, A paixão segundo G.H., [édition critique de Benedito Nunes], Madrid; Paris ; México ; Buenos Aires ; São Paulo ; Lima ; Guatemala ; San José de Costa Rica ; Santiago de Chile, ALCCA XX, 1997, p. 244. Nombreux critiques, notamment Olga Borelli et Benedito Nunes, se sont également intéressés à l'épiphanie chez Clarice Lispector. Cf. Olga BORELLI, A escritura de Clarice Lispector, Petrópolis, Vozes, 1979, p. 129-195 ; Benedito NUNES, O drama da linguagem, São Paulo, Ática, 1995.

4. Nous pouvons penser que la crise des personnages féminins monte in crescendo à mesure que nous avançons dans le recueil Laços de família. Avec cette même idée d'une amplification présente dans l'architecture du recueil, Cláudia Pazos Alonso se propose de relire le livre à partir des modulations de la thématique des relations humaines notamment en lien avec le pouvoir. Ces relations seraient représentées par les paires antagoniques homme/femme, dominateur/dominé, centre/périphérie. Cf. Cláudia PAZOS ALONSO, «Do centro e da periferia: uma releitura de Laços de família », Veredas, vol. 3, t. 1, (décembre 2000), p. 287-299.

5. «talvez seja o mais bem-humorado de toda a ficção de Clarice », Nádia Battela GOTLIB, op. cit., p. 322.

6. Clarice LISPECTOR, «Appendice : l'explication inutile ", Nouvelles [édition complète établie par Benjamin Moser], Paris, Éditions des femmes Antoinette Fouque, 2017, p. 468.

7. Ernani TERRA, «O enunciador em um conto de Clarice Lispector », Entretextos, vol. 20, n 1 (janvier-juillet 2020), p. 241-255. Cette préoccupation avec la norme européenne apparaît lors de l'étude des traductions de cette nouvelle vers l'espagnol: André Ramos García, «Decisiones traductoras y efectos en la lectura: estudio de dos traducciones al español de "Devaneio e embriaguez duma rapariga" de Clarice Lispector ", Cadernos de Tradução, vol. 40, n 1,2020 , p. 168-187 [disponible le 17/07/2020] <URL: https://periodicos.ufsc.br/index.php/traducao/ article/view/2175-7968.2020v40n1p168>.

8. Naomi LINDSTROM, «A Feminist Discourse Analysis of Clarice Lispector's "Daydreams of a Drunken Housewife" ", Latin American Literary Review, vol. 9, n¹9 (automne-hiver 1981), p. 7-16 [disponible le 13/05/2020] <URL : https://www.jstor.org/stable/20119271>.

9. Gabriela RUGGIERO NOR, Imagens de espelho em Clarice Lispector: entre reflexos e passagens [dissertação de Mestrado], São Paulo, USP/FFLCH, 2012.

10. Jean-Claude MIROIR et André Luís GOMES, «Poética e devaneio no processo criativo de Clarice Lispector », Fernanda Coutinho et Vera Moraes (org.), Clarices: uma homenagem (90 anos de nascimento, 50 anos de Laços de família), Fortaleza, Edições UFC, 2012, p. 35-55.

11. Il s'agit du mode de représentation le plus complexe de la vie intérieure d'un personnage dans un récit à la troisième personne, constitué par le « discours mental d'un personnage pris en charge par le discours du narrateur ", Dorrit COHN, La transparence intérieure, Paris, Seuil, 1989, p. 29. Pour une analyse des modes narratifs dans Laços de familia, cf. : Maria Luísa NUNES. " Modes in Clarice Lispector's Laços de familia: The rendering of Consciousness ", Luso-brazilian review, vol. 14, n², 1977, p. 174-184. 
12. Pour faciliter la lecture, nous indiquerons entre parenthèses directement les numéros des pages de la nouvelle étudiée dans l'édition suivante : Clarice LISPECTOR, « Devaneio e embriaguez duma rapariga ", Laços de família, Rio de Janeiro, 2009 [1960].

13. Moacyr GODOY MOREIRA, Linguagem e melancolia em Laços de família: histórias feitas de muitas histórias, [dissertação de mestrado], São Paulo, USP/FFLCH, 2007, p. 20.

14. Dicionário infopédia da Língua Portuguesa, Porto, Porto Editora, 2003-2020. [disponible le 19/07/2020] <URL : https://www.infopedia.pt/dicionarios/lingua-portuguesa/palestra>.

15. Dans le chapitre «Les moyens oniriques de la figuration », lié au concept d'identification, le fondateur de la psychanalyse précise que «tout rêve traite de la personne propre ", étant ainsi «absolument égoïste ». Le Moi peut ainsi se cacher derrière plusieurs personnes : «Je peux donc figurer mon Moi dans un rêve de manière multiple, une première fois directement, et ensuite par le moyen de l'identification avec des personnes étrangères. Avec plusieurs identifications de ce genre on peut condenser un matériau de pensées extraordinairement riche ", Sigmund FREUD, L'interprétation $d u$ rêve, traduction et présentation par Jean-Pierre Lefebvre, Paris, Seuil, 2010, p. 363-364.

16. Parmi les études consacrées à cette nouvelle que nous avons consultées, les seules à attribuer le prénom de Maria Quitéria à l'héroïne ou à considérer cette hypothèse à partir d'une véritable analyse sont: Jean-Claude MIROIR; André Luís GOMES. op.cit.; Gabriela RUGGIERO NOR, op.cit. soulèvent cette possibilité mais affirment que rien dans le texte permet d'en être sûr.

17. La référence à la folie ne se fait que par des allusions. À la fin de la nouvelle, le mari de Laura la trouve « sentada no sofá sem apoiar as costas, de novo alerta e tranquila como num trem. Que já partira. », Clarice LISPECTOR, «A imitação da rosa », Laços de família, op. cit., p. 53.

18. Clarice LISPECTOR, Liens de famille, traduit du brésilien par Jacques et Teresa Thiériot, Paris, Éditions des femmes Antoinette Fouque, 1989.

19. Op. cit.

20. Le terme a fait fortune après la publication de Madame Bovary (1857), de Gustave Flaubert, suite à la critique littéraire de Jules Barbey d'Aurevilly (1865), créateur du néologisme, et à l'emploi du terme en psychopathologie par Jules de Gaultier (1892), qui définit ce " mal de l'âme » de la manière suivante : "pouvoir départi à l'homme de se concevoir autre qu'il n'est ", Jules DE GAUlTIER, Le Bovarysme, suivi d'une étude de Per Buvik, Le Principe bovaryque, Paris, Presses de l'Université Paris-Sorbonne, 2006. Aujourd'hui, le champ littéraire s'est emparé également de la notion à partir de la figure de lectrice (cf. à ce sujet Delphine JAYOT, «Portrait(s) d'Emma : une femme "écrite" aux femmes décrites", Anne Herschberg Pierrot (dir.), Flaubert, éthique et esthétique, Saint-Denis, Presses universitaires de Vincennes p. 81-94), mais aussi d'un «bovarysme pantextuel», comme «affirmation du pouvoir du romanesque» qui, "autant que le donquichottisme, se confond avec la littérature, puisqu'on pourrait présenter l'intertexte comme le pouvoir départi au texte de se concevoir autre qu'il n'est...", Yvan LECLREC, «Bovarysme, histoire d'une notion ». Yvan Leclerc et Nicole Terrien. Le bovarysme et la littérature de langue anglaise, Mont-Saint-Aignan, Presses universitaires de Rouen et du Havre, 2004, p. 3-13 [disponible le 07/07/2020]

<URL : http://books.openedition.org/purh/7271>.

La définition de bovarysme qui nous intéresse ici est relative au rapport du personnage avec la société et à sa capacité de faire la part entre réel et fiction: «Le bovarysme se définit par l'insatisfaction affective ou sociale, nourrie d'imaginaire et romanesque, s'échappant dans des rêveries inatteignables et relevant de la capacité de l'homme à se concevoir autre qu'il n'est réellement. Certaines définitions mettent l'accent sur le rôle de la lecture de romans et de l'univers de fiction dans la production de cet état mental », Vassilis KAPSAMBELIS « Bovarysme ». Philippe Zawieja (dir.), Dictionnaire de la fatigue, Paris, Librairie Droz, 2016, p. 122. 
21. Rappelons que c'est ainsi que l'héroïne elle-même avait qualifié son mari lorsqu'il insistait pour la toucher avec un «carinho mais eficaz »: «- Ai, que não me maces! não me venhas a rondar como galo velho! » (p. 11, nous soulignons).

22. Robert ROWLAND, "Manuéis et Joaquins: a cultura brasileira e os portugueses ", Etnográfica, Vol. V (1), 2001, p. 157-172.

23. Le choix de Clarice Lispector de s'amuser en jouant avec la langue de l'ancien colonisateur, tout en le représentant à partir d'un stéréotype bas, ouvre une piste de lecture postcoloniale, à mettre en lien avec la représentation ambivalente des enjeux de pouvoir (mari/rapariga et mari/ riche commerçant) dans la nouvelle. Clarice Lispector semble ainsi «carnavaliser » le rôle du colonisateur tout en faisant appel à une représentation stéréotypée présente dans l'imaginaire brésilien. Boaventura de Sousa Santos rappelle que la représentation du Portugais immigré l'emporte sur celle du colonisateur, ce que le sociologue portugais explique par sa théorie d'une colonisation semipériphérique, dépendant surtout de l'Angleterre, et dont le résultat se cristallise dans l'image d'un «Prospéro calibanisé ». Cf. BOAVENTURA DE SOUSA SANTOS, «Entre Próspero e Caliban. Colonialismo, Pós-colonialismo e interidentidade ", Novos Estudos CEBRAP, n 66, julho 2003. Dans cette perspective postcoloniale, nous pouvons mettre «Devaneio e embriaguez duma rapariga » en lien avec la nouvelle « A menor mulher du mundo » publiée dans le même recueil.

24. Chez Clarice Lispector, l'épiphanie est souvent associée à la lumière et à la couleur blanche. Affonso Romano de Sant'Anna fait référence au » momento luminoso da epifania », op. cit, p. 244. Le critique associe l'épiphanie et le contraste blanc et noir à l'écriture :» $O$ texto é uma mancha contraditoriamente preta e branca que escorre como resultado de um massacre. Um massacre do ser. Do ser exposto à epifania. A epifania da escrita: traços escuros sobre um fundo branco », id.

25. Jean CHEVALIER et Alain GHEERBRANT, Dictionnaire des symboles, Paris, Robert Laffon, Jupiter, 1982, p. 855.

26. Regina Pontieri emploie justement l'image de la chair pour évoquer la thématique de l'altérité chez Clarice Lispector. La critique fait référence à cette « chair universel » dans laquelle tous les êtres communient. Cf. Regina PONTIERI, «Visões da alteridade: Clarice Lispector e Maurice Merleau-Ponty », Revista USP, nº 44 (déc-fév, 1999-2000), p. 330-334.

27. Il est intéressant de remarquer que cette opposition entre le dehors et le dedans, que nous avons maintes fois évoquée ici, rythme et structure aussi la nouvelle "Amor » à travers les déplacements d'Ana entre l'appartement et la rue, puis entre la rue et le Jardin Botanique. C'est la lecture que Paloma Vidal a fait de «Amor» lors de sa conférence Involontaires de la patrie: Clarice Lispector et les minorités, une lecture de la nouvelle "Amor», donnée à l'Université Bordeaux Montaigne, le 14 avril 2019.

28. Benedito Nunes analyse la nausée chez Clarice Lispector en établissant un lien évident avec La Nausée (1938), de Jean-Paul Sartre ; il compare l'expérience de Roquetin dans le Jardin Public avec celle d'Ana («Amor») dans le Jardin Botanique. Ce malaise subite et injustifié révèle, selon le critique, la contingence du sujet et l'absurde de ce qui l'entoure: «[s]inal do fascínio da consciência por aquilo que lhe é estranho e oposto", Benedito NUNES, op.cit. p.118. Chez Lispector, notamment dans A paixão segundo G.H. la nausée fait partie d'une extase mystique qui supprime le sens du monde. "A náusea é o modo extremo de descortínio contemplativo e silencioso que a fascinação das coisas provoca nos personagens de Clarice Lispector ", ibid., p. 122. 29. Valérie C. Lastinger, dans sa brève étude de l'humour dans les nouvelles de Laços de família, considère que l'effet comique chez Clarice Lispector s'explique par le décentrement de l'énoncé par rapport à la norme, révélant par-là l'absurde existentiel. Pour Lastinger, le passage où le riche commerçant «fait du pied» à la rapariga suivi de la mouche sur le décolleté de celle-ci constitue un cas typique du comique de situation. Valérie C. LASTINGER. "Humour in a new reading of Clarice Lispector », Hispania, 1989, p. 133. 
30. "um tanto embriagado ou bêbado ", Dicionário Houaiss da língua portuguesa, Rio de Janeiro, Editora Objetiva, 2001.

31. Le symbolisme de la lune se fait souvent par rapport à celui du soleil. Elle symbolise les rythmes biologiques, le temps qui passe et qu'elle permet de mesurer, la connaissance indirecte (du fait qu'elle n'est qu'un reflet du soleil), la fécondité car les animaux croissent et décroissent avec elle. En astrologie, la lune «symbolise le principe passif, mais fécond, la nuit, l'humidité, le subconscient, l'imagination, le psychisme, le rêve, la réceptivité, la femme et tout ce qui est instable, transitoire et influençable, par analogie avec son rôle astronomique de réflecteur de la lumière solaire », Jean CHEVALIER et Alain GHEERBRANT, op. cit., p. 593.

\section{RÉSUMÉS}

Dans cette étude sur "Devaneio e embriaguez duma rapariga", de Clarice Lispector, nous proposons d'analyser la première nouvelle du recueil Laços de família (1960) à partir de l'impression véhiculée par la critique d'une d'absence de prétention, d'une légèreté, voire d'une superficialité de l'expérience narrée qui expliquerait le peu d'intérêt suscité par ce texte. Nous cherchons à comprendre cette lecture de la nouvelle à partir de ses éléments textuels, tout en soulignant ce qui empêche ou remplace l'état de conscience aiguë de l'existence si souvent présent dans l'écriture de Clarice Lispector. Ainsi, on voit se dessiner une héroïne, dont l'origine portugaise ne nous semble pas hasardeuse, et qui personnifie de façon ambivalente les valeurs patriarcales de la famille brésilienne.

In this study on Clarice Lispector's "Devaneio e embriaguez duma rapariga", we propose to analyze the impression conveyed by the critic of an absence of pretension, a lightness, or even superficiality of the narrated experience that would explain the lack of interest aroused by this text. We seek to understand this reading of the story from its textual elements, highlighting what prevents or replaces the state of acute awareness of existence so often present in Clarice Lispector's writing. Thus, we see a heroine whose Portuguese origin does not seem hazardous to us, and who ambivalently personifies the patriarchal values of the Brazilian family.

\section{INDEX}

Keywords : Clarice Lispector, Devaneio e embriaguez duma rapariga, Laços de família, power, feminine

Mots-clés : Clarice Lispector, Devaneio e embriaguez duma rapariga, Laços de família, pouvoir, féminin

\section{AUTEUR}

\section{ILANA HEINEBERG}

Université Bordeaux Montaigne, GIRLUFI-AMERIBER 